\title{
A bio-inspired structure: conversing CdS to CdO for gas- sensing detection of acetone and diethyl ether
}

\author{
Xiangqian $\mathrm{Fu}^{1,2}$, Jinyun $\mathrm{Liu}^{2, *}$, Yuteng Wan ${ }^{1,2}$, Xiaoman Zhang $^{1,2}$, Xingjiu Huang ${ }^{2}$, Jinhuai Liu ${ }^{2, *}$ \\ ${ }^{1}$ Department of Chemistry, University of Science and Technology of China, Hefei 230026, PR China \\ ${ }^{2}$ Research Center for Biomimetic Functional Materials and Sensing Devices, Institute of Intelligent \\ Machines, Chinese Academy of Sciences, Hefei 230031, PR China \\ Corresponding authors: jyliu@iim.ac.cn and jhliu@iim.ac.cn
}

\begin{abstract}
:
A special CdO structure with a bio-inspired morphology was prepared by conversing CdS precursor under annealing treatment. The as-prepared leave-shaped $\mathrm{CdO}$ microstructures were further used for developing a sensitive gas sensor to detect acetone and diethyl ether. We find that the biomimetic structure can significantly improve the gas diffusion and adsorption of analytes in the sensing layer of gas sensor. During the gas-sensing process, the target gases can effectively diffuse through the $\mathrm{CdO}$ materials and adsorb onto the surface of them. The results show that presented gas sensor exhibits a fascinating gas-sensing performance with high response and short response time towards target gases. Our findings provide a potentially general strategy for developing sensitive gas sensor with enhanced diffusing and adsorbing properties towards analytes.
\end{abstract}

Key words: Biomimetic, semiconductor, gas sensor, sensitivity

\section{Introduction}

Bio-inspired materials have been of great interests due to their special physical and chemical properties which enable them to be potentially applied in a large variety of applications, e.g., electronic devices, lotus leaflike self-cleaning thin films, catalysts, etc [1-3]. Among various electronic devices, gas sensor as a type of important one has been widely desired in industrial manufacture, daily life and environmental monitoring $[4,5]$. Since the past decade, developing sensitive gas sensors with high response, short response and recovery times for applications has become a great challenge depending on the rapid development of modern technology. In recent years, many efforts have been contributed to improve the sensing performance of gas sensors. However, the enhancement on gas diffusion and adsorption/desorption which greatly influence the contact efficiency between gas analytes and sensing materials has been rarely focused on.

Seen from the leaves in nature, it can be found that almost all the leaves can adequately contact with the surroundings including sunlight, water vapor, and air. Therefore, preparing novel gas-sensing materials with such a special leaflike structure would be a significant strategy to effectively improve the contact between sensing materials and target gas molecules. Herein, we report a novel gas sensor based on special leave-shaped $\mathrm{CdO}$ microstructures which exhibits a sensitive performance towards acetone and diethyl ether. The biomimetic $\mathrm{CdO}$ microstructures were prepared by annealing leave-shaped CdS precursors which were synthesized via a hydrothermal route.

\section{Results and discussion}

The typical morphology of the as-synthesized precursors and final products are shown in Fig. 1. As can be seen from Fig. $1 a$ and $b$, the precursors exhibit a special profile of threedimensionally leave-shaped structure. The length and thickness of each leave are ca. $3 \mu \mathrm{m}$ and $50 \mathrm{~nm}$, respectively. After the thermal treatment towards the precursors at $500^{\circ} \mathrm{C}$ for $1 \mathrm{~h}$ in air, the samples keep a relatively similar leave-shaped morphology, as shown in Fig. 1c and d. However, some parts of the microstructure are ruined. Some particles are formed accompanying with the reduction of each leave. It would be ascribed to the conversion of phases from $\mathrm{CdS}$ to $\mathrm{CdO}$, which can be supported by the XRD patterns (Fig. 1e and $f$ ). Seen from Fig. $1 e$ and $f$, it can be confirmed that the precursors and products are $\mathrm{CdS}$ and $\mathrm{CdO}$, respectively. It is indicated that the $S$ atoms in CdS precursors have been replaced by $\mathrm{O}$ atoms under annealing treatment in air, resulting in the formation of $\mathrm{CdO}$ crystals. 

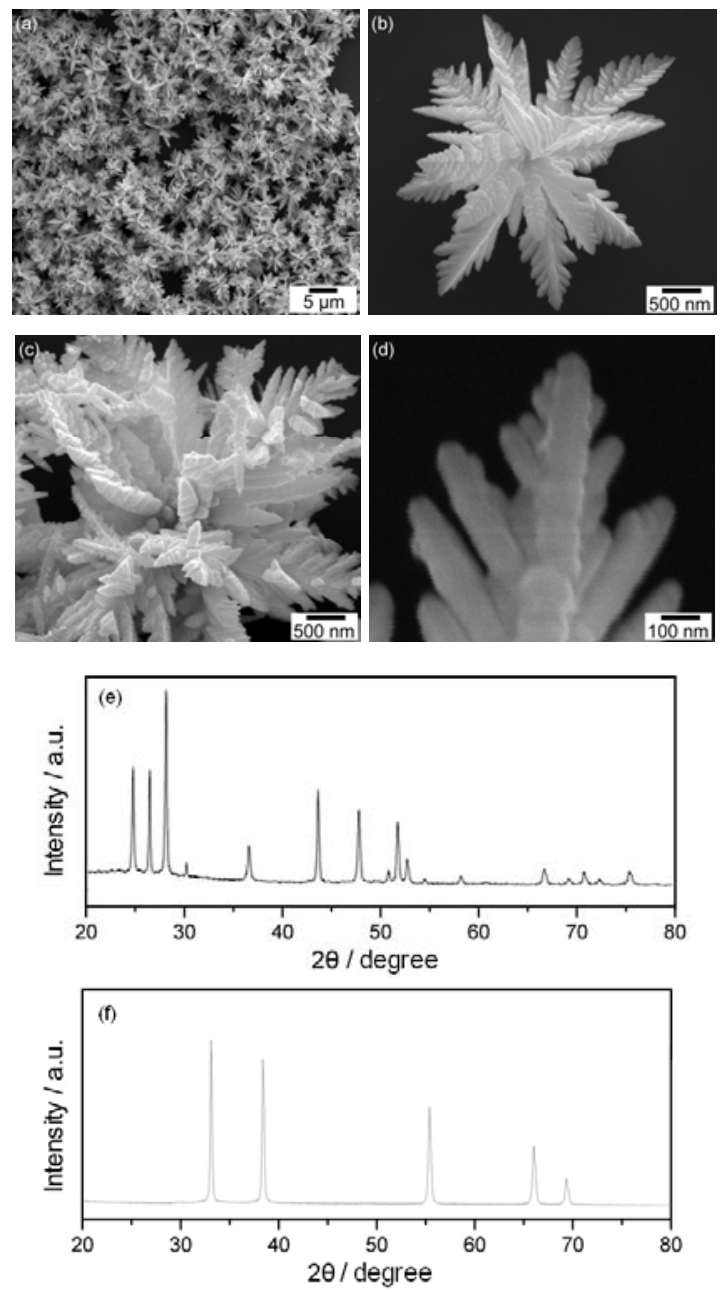

Fig. 1. FESEM images of (a), (b) the as-synthesized CdS precursors and (c), (d) the CdO prepared by annealing precursors in air; (e) and ( $f$ ) are corresponding XRD patterns, respectively.

In order to further investigate the phaseconversing behavior, the CdS precursors were also annealed under a relatively lower $\left(400^{\circ} \mathrm{C}\right)$ and higher temperature $\left(550{ }^{\circ} \mathrm{C}\right)$ for $2 \mathrm{~h}$, respectively. Fig. 2 shows the FESEM images of the as-obtained samples. As can be seen, the samples remain a completely leave-shaped morphology under a low temperature in annealing process. However, we find that the phase conversion from $\mathrm{CdS}$ to $\mathrm{CdO}$ cannot be achieved completely at such a low temperature (the XRD pattern is not shown here). When the annealing conditions change to be at a temperature of $550{ }^{\circ} \mathrm{C}$ for a time of $2 \mathrm{~h}$, only particles can be observed. All the leave-shaped morphology disappears. So, by combining with the leave-shaped morphology and the complete phase conversion, the $\mathrm{CdO}$ samples obtained after a relative mild annealing condition were used to fabricate gas sensor for gas-sensing measurements.
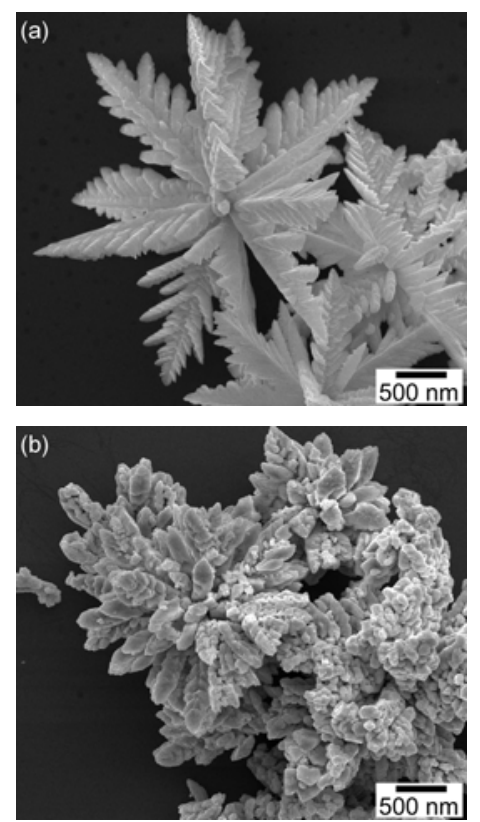

Fig. 2. FESEM images of the samples prepared by annealing CdS precursors at (a) 400 and (b) $550{ }^{\circ} \mathrm{C}$ for $2 h$.

Fig.3 shows the gas-sensing performance of the sensor based on the leave-shaped $\mathrm{CdO}$ microstructures towards acetone and diethyl ether. Seen from Fig. $3 a$ and $b$, the currents increase rapidly when the target gases were injected into the detecting chamber, indicating that the sensor is sensitive towards analytes. The gas-sensing mechanism can be ascribed to the typical sensing behavior of semiconductors [6]. In air atmosphere, $\mathrm{O}_{2}$ is adsorbed on the surface of $\mathrm{CdO}$ microstructures. It will capture and react with electrons from $\mathrm{CdO}$ to produce negative oxygen species, including $\mathrm{O}_{2}^{-}, \mathrm{O}^{-}$, and $\mathrm{O}^{2-}$. And then, a depletion layer is formed, resulting in a high resistance of $\mathrm{CdO}$ materials. When the $\mathrm{CdO}$ microstructure-based gas sensor is exposed to reducing gases like acetone and diethyl ether, some chemical reactions between oxygen species and gas analytes will take place, leading to the release of electrons back to $\mathrm{CdO}$ and thereby increasing the current of circuit.

The gas response calculated from the real-time sensing curves is shown in Fig. 3c, which can be a support for such a sensitive gas-sensing property of the presented gas sensor. Even towards the analytes at a concentration of 0.5 ppm, we find that the sensor can exhibit remarkable gas responses. The response and recovery times are presented in Table 1 . The short response time indicates that the biomimetic microstructures can significantly enhance the surface contact between target molecules and sensing materials. The special leave-shaped structure not only be 
advantageous for excellent connect between each $\mathrm{CdO}$ architecture, but also can keep a relatively large remained space for gas diffusion and adsorption because of the support of leaveshaped morphology. It plays a role similar to the leaves of plant in nature for contacting the surroundings effectively, like sunlight, air, and water vapor.
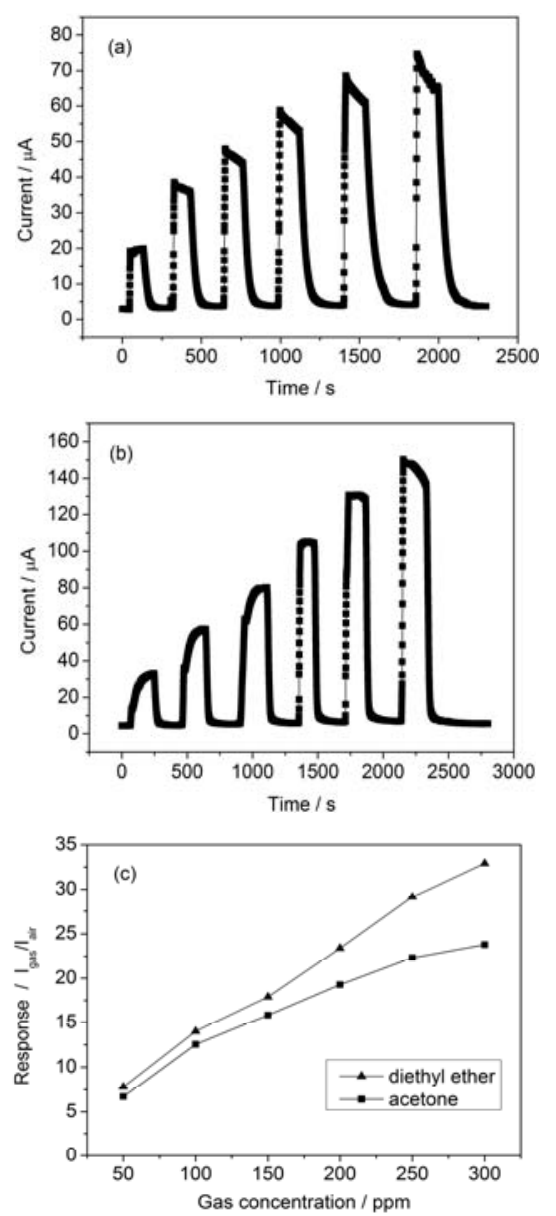

Fig. 3. Real-time gas-sensing curves of the sensor towards (a) acetone and (b) diethyl ether; (c) is the calculated values of response.

Tab. 1: The response and recovery times of the gas sensor based on CdO microstructures.

\begin{tabular}{|c|c|c|c|c|}
\hline \multirow{2}{*}{$C_{\text {gas }} / \mathrm{ppm}$} & \multicolumn{2}{|c|}{ Acetone } & \multicolumn{2}{c|}{ Diethyl ether } \\
\cline { 2 - 5 } & $t_{\text {res }} / \mathrm{s}$ & $t_{\text {red }} / \mathrm{s}$ & $t_{\text {res }} / \mathrm{s}$ & $t_{\text {red }} / \mathrm{s}$ \\
\hline 50 & 4.6 & 36.5 & 8.9 & 18.3 \\
\hline 100 & 8.9 & 43.1 & 10.5 & 22.8 \\
\hline 150 & 10.4 & 45.9 & 29.8 & 24.4 \\
\hline 200 & 10.3 & 55.1 & 24.3 & 26.4 \\
\hline 250 & 12.6 & 76.8 & 22.8 & 21.5 \\
\hline 300 & 10.5 & 68.3 & 17.1 & 23.8 \\
\hline
\end{tabular}

\section{Conclusions}

In summary, a bio-inspired $\mathrm{CdO}$ microstructure which possesses a leave-shaped morphology prepared by conversing CdS precursor was reported for gas-sensing application. In the process of annealing conversion, it is found that neither too low nor too high temperature in annealing process is advantageous to achieve a leave-shaped structure and a complete phase conversion. The gas-sensing results show that the gas sensor based on the special $\mathrm{CdO}$ microstructures is sensitive towards analytes including acetone and diethyl ether. The enhancing mechanism could be ascribed to the special leave-shaped structure which can provide a proper condition for the improvement of gas diffusion.

\section{Acknowledgements}

This work was financially supported by the "973" State Key Project of Fundamental Research for Nanoscience and Nanotechnology (2011CB933700), the National Natural Science Foundation of China $(51002157,61071054$, 61104205, 21177131, and 90923033), and the One Hundred Person Project of the Chinese Academy of Sciences.

\section{References}

[1] P. Domachuk, K. Tsioris, F. G. Omenetto, D. L. Kaplan, Bio-microfluidics: Biomaterials and Biomimetic Designs, Advanced Materials 22, 249-260 (2010); doi: 10.1002/adma.200900821

[2] V. Abbate, A. R. Bassindale, K. F. Brandstadt, P. G. Taylor, Biomimetic Batalysis at Silicon Centre Using Molecularly Imprinted Polymers, Journal of Catalysis 284, 68-76 (2011); doi: 10.1016/j.jcat.2011.08.019

[3] L. P. Wen, X. Hou, Y. Tian, J. Zhai, L. Jiang, Bioinspired Photoelectric Conversion Based on Smart-Gating Nanochannels, Advanced Functional Materials 20, 2636-2642 (2010); doi: 10.1002/adfm.201000239

[4] I. Sayago, M. J. Fernandez, J. L. Fontecha, M. C. Horrillo, C. Vera, I. Obieta, I. Bustero, Surface Acoustic Wave Gas Sensors Based on Polyisobutylene and Carbon Nanotube Composites, Sensors and Actuators B-Chemical 156,1-5 (2011); doi: 10.1016/j.snb.2011.04.047

[5] R. B. Birajadar, A. Ghosh, A. Ghule, F. Singh, R. Sharma, Enhancement of LPG Sensing Properties in Nanocrystalline Zinc Oxide Thin Film by High Electronic Excitation, Sensors and Actuators B-Chemical 160, 1050-1055 (2011); doi: 10.1016/j.snb.2011.09.024

[6] X. L. Gou, G. X. Wang, J. S. Park, H. Liu, J. Yang, Monodisperse Hematite Porous Nanospheres: Synthesis, Characterization, and Applications for Gas Sensors, Nanotechnology 19, 125606 (2008); doi:10.1088/0957$4484 / 19 / 12 / 125606$ 